\title{
Measuring and Improving the Quality of Hospital Language Services: Insights from the Speaking Together Collaborative
}

\author{
Marsha Regenstein, PhD MCP \\ Department of Health Policy, The George Washington University Medical Center, Washington, DC, USA.
}

J Gen Intern Med 22(Suppl 2):356-9

DOI: $10.1007 / \mathrm{s} 11606-007-0358-2$

(c) Society of General Internal Medicine 2007

\begin{abstract}
T $\mathrm{n}$ 2006, the Robert Wood Johnson Foundation launched Speaking Together: National Language Services Network, an initiative designed to improve the quality and availability of language services in US hospitals. Speaking Together works with hospitals that were selected through a competitive application process with multiple levels of review. The selection process targeted hospitals with substantial numbers of limited English-proficient (LEP) patients and well-established language programs that could complete a complex project with rigorous data collection requirements. The result is a group of 10 hospitals (Table 1) whose language services are more robust than the average hospital and that bring enthusiasm to the process of performance improvement in language services, dedication to working collaboratively through interdisciplinary project teams, and strong commitment from senior leadership to support their efforts.

In this Commentary, I offer personal insights from my role as Director of the National Program Office for Speaking Together. First, I discuss the process of developing and some of the challenges of implementing performance measures for hospital language services. Then, I offer some lessons learned from my own "field experience" with the Speaking Together project thus far about what I consider key elements for creating and implementing effective language services programs.
\end{abstract}

\section{GETTING STARTED: DEFINING PERFORMANCE MEASURES FOR LANGUAGE SERVICES}

For months leading up to the official launch of Speaking Together, I visited some hospitals and spoke with staff and clinicians at many others, trying to understand the operational aspects of the delivery of language services, the barriers to accessing care, the financial challenges associated with operating various components of language services, and the organizational and cultural issues that drive elements of the service. Getting the details was fairly straightforward, and I learned that there is an emerging "science" to the delivery of language services. Some practices clearly work better than others, and years of trial and error at many different places across the country provide practical lessons that will save time and money for newly designed programs. For example:

- using patient registration systems that link directly to interpreter scheduling systems appears to decrease wait times for interpreters and allows interpreter services managers to anticipate resource needs across scheduled and unscheduled visits;

- relying on rosters of self-identified bilingual staff and providers as the principal vehicle for addressing the needs of LEP patients will result in the majority of encounters being handled through the use of family and friends;

- dedicating interpreter time to high-volume locations, or blocking clinic time for patients speaking certain languages, can increase interpreter efficiency and reduce patient wait times.

Early site visits and interviews with field experts also uncovered a clear understanding of what it means to be a qualified interpreter. Formal training in medical interpreting coupled with assessment for language fluency were commonly considered key attributes of high-quality interpreters. However, there was less clarity in the definition of quality, as it pertained to actually delivering those services.

The closest "measure" of quality in the delivery of language services appeared to be an assessment of volume: higher volume programs (especially those using trained interpreters) were cited as models for quality in service delivery. The volume of interpreter encounters was a number that was well known throughout the hospital, yet even at these "high-performing hospitals," no one knew the percent of LEP patients who came to their hospitals and actually received language services while in their care. Nor was there agreement on what would constitute appropriate services for these patients. While a few hospitals set goals for full and timely access to high-quality interpreters for all LEP patients, none had attempted the ambitious task of designing processes to determine how well they were meeting their goal of reaching every LEP patient. And while several noteworthy hospitals paved the way for quality improvement, for example by tracking the timeliness of care, their measurements were limited to those patients who used their services.

Speaking Together developed performance measures for language services primarily to address this gap in knowledge. We used a staged process over a 10-month period to identify measures that could be used in a quality improvement hospital collaborative. We found the 6 domains of quality identified by the Institute of Medicine-safety, timeliness, efficiency, effectiveness, equity, and patient-centeredness ${ }^{1}$ - to 
Table 1. Speaking Together Hospitals

\begin{tabular}{l}
\hline Hospitals \\
\hline Bellevue Hospital Center, New York, NY \\
Cambridge Health Alliance, Cambridge, MA \\
Children's Hospital and Regional Medical Center, Seattle, WA \\
Hennepin County Medical Center, Minneapolis, MN \\
Phoenix Children's Hospital, Phoenix, AZ \\
Regions Hospital, St. Paul, MN \\
UMass Memorial Health Care, Worcester, MA \\
UC Davis Health System, Sacramento, CA \\
University of Michigan Health System, Ann Arbor, MI \\
University of Rochester Medical Center, Rochester, NY \\
\hline
\end{tabular}

be a useful framework for defining quality in language services. We used these domains to categorize attributes of quality in a comprehensive review of the literature that we conducted to develop an evidence base for the performance measures. The evidence from the published literature was limited yet crystal clear on one key principle: in encounters where providers and patients did not speak the same language, quality of care (according to many quality-related measures) was nearly always better when a trained interpreter was present compared to care delivered without a trained interpreter. This finding served as a guiding principle for the development of our performance improvement work.

We supplemented the literature review with dozens of interviews with researchers, physicians, nurses, hospital leadership and service line managers, and interpreter services directors to gather information about the practical implications of various aspects of quality on language services and other hospital operations. We used a framework for organizational change developed by Nerenz and Neil ${ }^{2}$. We drafted 10 performance measures and assembled an expert panel, composed of directors of hospital interpreter services programs as well as physicians and managers of ambulatory services, to evaluate each measure. The expert panel reviewed the 10 measures, evaluated each according to its importance to quality, feasibility in terms of data collection, clarity, and accuracy of the description. The expert panel narrowed the proposed list of 10 measures to 5 . We then piloted the measures at 2 non-Speaking Together hospitals. The 5 measures being used in the Speaking Together quality improvement collaborative are:

- ST 1: The percent of patients who have been screened for their preferred spoken language

- ST 2: The percent of LEP patients receiving initial assessment and discharge instructions from assessed and trained interpreters or from bilingual providers assessed for language proficiency

- ST 3: The percent of encounters where the patient wait time for an interpreter is $15 \mathrm{~min}$ or less

- ST 4: The percent of time interpreters spend providing medical interpretation in clinical encounters with patients

- ST 5: The percent of encounters interpreters wait less than 10 minutes to provide interpreter services to provider and patient. $^{3}$

Central to the concept of quality in Speaking Together is the notion that hospitals must move away from thinking about their volumes in terms of interpreter encounters or minutes of telephone interpreting. Instead, hospitals must construct language services systems to address their patients' needs and ultimately frame quality around two critical questions:

1. Who among your patients could benefit from language services (either directly through care from a bilingual provider or indirectly through interpretation via some third party modality)?

2. Have these patients actually received language services?

The Speaking Together hospitals are among the first in the country to begin to answer these questions, and the process is not as clean and simple as one might hope. The hospitals submit monthly data reports to the Speaking Together National Program Office on just 5 performance measures, yet they require enormous effort on the part of the hospitals' staff and operations. Hospitals are required to screen all patients for preferred language. That means asking even patients who are speaking English, or who have a family member or friend speak for them, in what language they prefer to receive their health care. The goal here is to stop determining need for language services based on whether patients say they want an interpreter, as too often, patients decline if they are accompanied by someone who speaks both their native language and English or if they perceive the wait for an interpreter to be too long.

Most of the hospitals had screening systems in place before the project and are doing well on our first screening measure (ST 1). They have much more difficulty responding to the second measure, which requires them to identify whether the patient actually received care in the preferred language. One of the biggest challenges is accounting for the use of bilingual providers who help meet the needs of LEP patients as both direct providers and ad hoc interpreters.

Given the numerous times a patient interacts with a hospital system during the inpatient stay and the difficulty in collecting data on interactions, Speaking Together chose to focus on 2 key components of inpatient care: initial assessment and discharge. Hospitals in the collaborative must determine whether each LEP patient received adequate language services during both of these two interactions. In cases where patients receive language services during just one of these interactions, the hospital records that the LEP patient did not receive adequate language services. The goal of the Speaking Together hospitals-as should be the goal of all hospitals across the country-is that all LEP patients receive adequate language services during those (and indeed many other) critical interactions that depend so heavily on effective communication. This can be achieved in a variety of ways (e.g., in-person interpreters, telephones, video), but cannot be replaced by untrained interpreters, providers who fall short in their language proficiency, or family and friends.

Speaking Together hospitals are testing new ways to track which patients needing language services actually receive them but are struggling with ways to identify providers who can be considered bilingual. With proper assessment for fluency and training for medical interpreting, considerable resources could exist in-house to address language needs. But finding them, deploying them, addressing the labor-related issues associated with adding new job responsibilities to staff position descriptions, and back-filling the temporary workflow gaps created when responding to a call for help from language services will require careful planning, with input from all parties involved. 
Hospitals are also tracking performance on the timeliness and efficiency of language services with the hopes of improving responsiveness to patients and providers and making the most of limited resources. Our experiences so far have shown that hospitals can indeed engage in meaningful quality improvement and integrate language services within the corporate culture of the complex health system environment.

\section{ELEMENTS OF EFFECTIVE LANGUAGE SERVICES: NOTES FROM THE FIELD}

My experiences with Speaking Together hospitals and others across the country have led me to the following conclusions about creating and implementing effective language services programs:

Just Because You Build It Does Not Mean They Will Come. So much of the conversation about language services concentrates on the benefits associated with interpreters and other modes of language services delivery. Yet merely securing the services does not promise that the patients who need them will get them. Without demand from physicians and nurses, the supply of language services will go untapped or underutilized. In many hospitals and health care organizations across the country, interpreters spend precious hours sitting idle, or taking on responsibilities that could be completed by someone without their skills in medical interpretation, because the health care professionals who could benefit most from their help do not demand that the service be provided. So why do physicians and nurses not push the demand side of the equation?

\section{Educating Providers Is Important, But Making the Service Easy} to Access Is Critical. While language services are a complex addition to the health care encounter, they are absolutely essential for every dimension of quality. Many interpreter services department managers have spoken to me about "making the quality case" for language services to their clinical and administrative staffs, placing their hopes on the link between language services and quality to push demand for language services. Unfortunately, anecdotal evidence suggests that while educational efforts can produce results, substantial improvements come about when the "logistics" associated with language services are simplified. In other words, the easier it is for physicians and nurses to obtain the language service, the more likely they will include it as routine practice. And the more likely they include it as routine practice, the less satisfied they will be without them.

The More Senior Leadership Pays Attention to the Performance of Language Services, the Greater the Likelihood that Patients Will Reap the Benefits. As the visibility of language services programs in Speaking Together hospitals has grown over the life of the project, so too has its accountability. Several chief executives now report to their hospital boards about the quality of the language services program; in one hospital, the interpreter services director has been made a permanent member of the system-wide quality improvement committee. Speaking Together teams report smoother sailing when negotiating changes to information technology systems, requesting equipment upgrades or promoting greater integration with clinical staffs with the chief executive and other senior management on record supporting these activities and linking them directly with enhancements in patient care.

Involving Providers from Different Sectors of the Health Care Organization in Decisions regarding Language Services Is Vital. I have enormous respect for medical interpreters and the language services workforce, and I believe their contributions to the quality of health care delivery are incalculable. We must remain focused, however, on the 2 parties that are the clients of language services-the patient and the provider. Good language services should be easy to access and seamless to the health care experience. They are the or more "sound system" that makes the orchestra's playing come alive. Without their technical skills, the music could not flow from the artists to the audience. But a stateof-the-art sound system is of little use if it cannot adapt to the varying needs of the orchestra. In the same vein, creating or expanding the language services component of an organization, setting performance standards, and identifying areas for improvement should be built on input from the various clients in the health care organization, whose work the language services are intended to facilitate. Each dimension of quality may be interpreted differently by various departments in the hospital. For example, 15-minute average wait times for an interpretation may be fine for the primary care clinic but unacceptable in the emergency department. In-person interpretation may be the preferred mode for genetic counseling, but not always necessary for post-surgical follow-ups.

The following illustrates the real-world impact of providing language services without input from the client. In one hospital with a large well-established language services program, "bad blood" between the cardiac testing department and interpreter services was interfering with effective services for LEP patients. The interpreters hated seeing patients for nuclear stress testing because the nurse manager for the cardiology department always seemed disgruntled and angry when the interpreter arrived, especially if the interpreter was late for the encounter. It was only after the interpreter confronted the nurse manager with her rudeness and asked pointedly why the lateness was such a problem that she learned that the medication used for the nuclear stress test lost effectiveness after a certain period of time. If the patient needed to be re-dosed, there was an additional cost of $\$ 800$, which came out of cardiology's budget. Protocols in the hospital were subsequently changed to ensure that requests for interpreters for patients receiving nuclear stress tests were handled expeditiously. The change to the interpreter appointment system required relatively minor modification yet resulted in a substantial change in relations between the two departments and had the added benefits of eliminating wasteful and costly practices.

Do Not Leave the Patient Out of the Discussion. Checking in with patients about how well language services are meeting their needs should be a routine part of quality assessment. Satisfaction surveys provide some input on patients' perceptions regarding communication via interpreters but are rarely sufficiently detailed to identify ways to improve services, especially if they differ across language groups. Holding informal focus groups with LEP patients can identify barriers to accessing services as well as the aspects of the service that they value most. For example, 
continuity of interpreter may be a value that outstrips timeliness for some patients. Likewise, patients may prefer telephone or inperson interpretation for different types of encounters, but may not be aware that they can voice this preference when they present for care.

In the end, the abiding lesson is that change is hard, and it is no more or less hard when it comes to language services. The good news from the Speaking Together program is that change is possible and in fact occurring at hospitals across the country. And the even better news is that patients will be the winners at the end of the day.

Acknowledgments: Support for the work described in this article comes from The Robert Wood Johnson Foundation.

Funding: Dr. Regenstein is supported by a grant from the Robert Wood Johnson Foundation, number 055661. The views expressed here are those of the author and should not be attributed to the Robert Wood Johnson Foundation or its directors, officers, or staff.

Corresponding Author: Marsha Regenstein, PhD MCP; Department of Health Policy, The George Washington University Medical Center, $2121 \mathrm{~K}$ Street, NW, Suite 210, Washington, DC 20037, USA (e-mail: marshar@gwu.edu).

\section{REFERENCES}

1. Institute of Medicine. or more "Crossing the Quality Chasm: A New Health System for the 21st Century," Washington, DC: National Academies Press, 2001.

2. Nerenz DR, Neil N. Performance measures for health care systems (commissioned paper for the Center for Health Management Research); May 2001. Available at: http://www.hret.org/hret/programs/chmr/pls/pls10.html. Accessed August 26, 2007.

3. Speaking Together: National Language Services Network. Performance improvement measures. Available at: http://www.speakingtogether.org/measures. Accessed August 26, 2007 